\title{
Associations between neutrophil/lymphocyte ratio, platelet/lymphocyte ratio, albuminuria and uric acid and the estimated glomerular filtration rate in hypertensive patients with chronic kidney disease stages $1-3$
}

\author{
Nurhayat Ozkan Sevencan, Aysegul Ertinmaz Ozkan
}

Department of Internal Medicine, Medical Faculty, University of Karabuk, Karabuk, Turkey

Submitted: 3 March 2018

Accepted: 20 May 2018

Arch Med Sci 2019; 15 (5): 1232-1239

DOI: https://doi.org/10.5114/aoms.2018.76262

Copyright (c) 2018 Termedia \& Banach

\section{Abstract}

Introduction: The neutrophil/lymphocyte ratio (NLR), platelet/lymphocyte ratio (PLR), albuminuria and uric acid are known to be independent predictors of hypertension and cardiovascular mortality. However, to date, no study has been conducted describing the relationships between the NLR, PLR and estimated glomerular filtration rate (eGFR) in hypertensive patients with chronic kidney disease (CKD) who do not require renal replacement therapy.

Material and methods: This prospective study included 271 patients with essential hypertension and eGFR $\geq 30 \mathrm{ml} / \mathrm{min} / 1.73 \mathrm{~m}^{2}$. The patients were divided into two groups: those with CKD stages 1 and 2 and those with stage 3 . We used the complete blood count to calculate the NLR and PLR, and we measured the albuminuria and uric acid levels. Then, we studied their associations with the eGFR and their potential uses as independent risk factors for renal damage.

Results: The NLR, albuminuria and uric acid were higher in patients with CKD stage 3 than in those with stages 1 and $2(p=0.013, p=0.001$ and $p=0.001$, respectively). However, no significant difference was detected in the PLR. In stage 3 patients, albuminuria and uric acid were found to be independent risk factors affecting the eGFR $(p=0.042$ and $p=0.001$, respectively). However, the effects of the NLR and PLR on the eGFR were not significant ( $p=0.104$ and $p=0.578$, respectively).

Conclusions: The NLR, similar to albuminuria and uric acid, the NLR was found to be a specific marker for CKD stage 3 patients. However, the NLR and PLR did not act as independent risk factors affecting the eGFR.

Key words: neutrophil/lymphocyte ratio, platelet/lymphocyte ratio, uric acid, albuminuria, estimated glomerular filtration rate, chronic kidney disease.

\section{Introduction}

Essential hypertension, also known as primary hypertension, refers to a condition of high blood pressure without any secondary cause, and it may result in cardiovascular disease. The aetiology of essential hypertension is multifactorial, with inflammation being an important factor in its pathogen-

\author{
Corresponding author: \\ Nurhayat Ozkan Sevencan \\ $\mathrm{PhD}$ \\ Department \\ of Internal Medicine \\ Medical Faculty \\ University of Karabuk \\ 78100 Karabuk, Turkey \\ Phone/fax: +90 5053961458 \\ E-mail: dr_nurhayat@ \\ hotmail.com
}


esis [1]. Studies have shown the significant role that inflammation plays in the onset and progression of cardiovascular and renal diseases [2-6].

An increased count of leukocytes (and their subtypes) is a hallmark of the inflammatory process, and it is closely associated with cardiovascular risk [7]. The neutrophil/lymphocyte ratio (NLR), which is derived from the leukocyte count, has been investigated with regard to cardiovascular risk, and it was found to be an important inflammation marker [8-11]. Similarly, the platelet/lymphocyte ratio (PLR) has been shown to be associated with important cardiovascular outcomes. This is attributed to the increased platelet activation observed in the onset and progression of atherosclerosis [12]. Additionally, serum uric acid and albuminuria, which are independent determinants of a future increase in the systolic blood pressure, may be considered among the independent risk factors for hypertension, as well as cardiovascular and renal damage [13-18].

Although the NLR and PLR have been evaluated as cardiovascular risk factors in many studies, research regarding their associations with renal damage and the estimated glomerular filtration rate (eGFR) is limited [19]. Moreover, to the best of our knowledge, there have been no studies performed in patients with chronic kidney disease (CKD) who do not require renal replacement therapy.

Therefore, in the present study, we investigated the associations between the NLR, PLR, albuminuria and serum uric acid and the eGFR. In addition, we investigated whether the NLR and PLR were independent predictors of renal damage in patients with essential hypertension and CKD stages 1-3.

\section{Material and methods}

\section{Design and patients}

This prospective study included 271 patients over 40 years old with essential hypertension and eGFRs $\geq 30 \mathrm{ml} / \mathrm{min} / 1.73 \mathrm{~m}^{2}$ who were admitted to our internal medicine outpatient clinic between June 2017 and January 2018. All the patients were evaluated for the presence of risk factors, including secondary hypertension, hyperlipidaemia, diabetes mellitus, renal, cardiovascular and cerebrovascular diseases and smoking.

\section{Blood pressure measurements}

Hypertension was defined as systolic blood pressure (SBP) $\geq 140 \mathrm{~mm} \mathrm{Hg}$ and/or diastolic blood pressure $(D B P) \geq 90 \mathrm{~mm} \mathrm{Hg}$, previously diagnosed hypertension, or use of any antihypertensive medications. The arterial BP of each patient was measured using a standardized protocol. Each patient remained at rest for at least $10 \mathrm{~min}$ in a seated position, and their arms were supported at the level of the heart. All of the patients wore light clothing (no tight clothing constricting the arms), and they were kept in optimal room conditions. At least two blood pressure measurements (mean: $2.2 \pm 0.5$ measurements) at 5-min intervals were taken from each patient from both the arms in mild flexion using an aneroid sphygmomanometer (Perfect Aneroid sphygmomanometer; ERKA, Bad Tölz, Germany). The mean of the two readings from the higher arm side was accepted as the BP. If there was more than a $5 \mathrm{~mm} \mathrm{Hg}$ difference between the first and second readings, additional (one or two) readings were obtained, and the average of these multiple readings was used. The SBP was accepted as the first Korotkoff sound phase, while the DBP was the fifth phase (disappearance of sounds) to the nearest $2 \mathrm{~mm} \mathrm{Hg}$. Three different cuff sizes were used in these patients according to their arm circumferences (small adult $=12 \times$ $18 \mathrm{~cm}$, standard adult $=12 \times 26 \mathrm{~cm}$ and large adult $=12 \times 40 \mathrm{~cm}$ ) [20]. The aneroid sphygmomanometers were calibrated using a standardized mercury column manometer.

\section{Exclusion criteria}

Patients with diabetes mellitus, hematopoietic system disorders, histories of malignancy and/or chemotherapy treatment, signs of accompanying infectious diseases, leukocyte disorders (such as an acute infection or chronic inflammatory status), histories of secondary hypertension, cardiac failure and chronic liver disease, and those who had used glucocorticoid therapy within the last 3 months were excluded from the study. Comprehensive medical history was taken from the patients to exclude secondary hypertension. All of the patients underwent renal artery Doppler ultrasonography (USG), and the renal resistive indexes were measured to exclude secondary hypertension. We also scanned all of the patients for adrenal adenomas during the USG.

Each of the patients signed an informed consent form, and the study protocol was approved by the local ethics committee.

\section{Laboratory assessments}

Upon admittance, a complete blood count, including the leukocytes, neutrophils, lymphocytes and platelets, and the blood urea nitrogen, creatinine and uric acid were evaluated using venous blood. Diabetes mellitus was defined as fasting plasma glucose levels more than $126 \mathrm{mg} / \mathrm{dl}$ in multiple measurements. Venous blood was taken from the patients in the morning after $8 \mathrm{~h}$ of fasting. Previously diagnosed diabetes mellitus and/ or use of antidiabetic medications such as oral anti-diabetic agents or insulin were considered to 
be DM as well. The microalbumin was evaluated in the spot urine collected from the patients. The NLR was calculated from the ratio of the neutrophil to lymphocyte counts and the PLR was calculated from the ratio of the platelet to lymphocyte counts. The eGFR was calculated using the Chronic Kidney Disease Epidemiology Collaboration (CKD-EPI) equation [21]. The patients were divided into two groups: those with an eGFR $\geq 60 \mathrm{ml} /$ $\mathrm{min} / 1.73 \mathrm{~m}^{2}$ (CKD stages 1 and 2) and those with $60>\mathrm{eGFR} \geq 30 \mathrm{ml} / \mathrm{min} / 1.73 \mathrm{~m}^{2}$ (CKD stage 3 ).

\section{Statistical analysis}

The Number Cruncher Statistical System 2007 (NCSS Statistical Software, Kaysville, UT, USA) was used for the statistical analyses. We used descriptive statistical methods (mean, standard deviation, frequency, percentage, minimum and maximum) to assess the study data. Additionally, the normality of distribution of the quantitative data was tested using the Shapiro-Wilk test and graphical examinations. For the normally distributed quantitative variables, a one-way analysis of variance was used for the comparison of more than two groups. Bonferroni-corrected post-hoc tests were used for the paired comparisons. Pearson's correlation test was used to reveal a linear association between eGFRs, albuminuria and serum uric acid levels. A logistic regression analysis was used to assess the risk factors affecting the eGFR. To determine the cut-offs for the parameters, diagnostic tests (sensitivity, specificity, positive predictive value (PPV), negative predictive value (NPV) and accuracy) and receiver operating characteristic (ROC) curve analyses were used. The comparisons of the areas under the ROC curve were performed using the DeLong method. The statistical significance was set at $p<0.05$.

\section{Results}

The present study included 185 (68.3\%) females and 86 (31.7\%) males, and their mean age was $63.94 \pm 11.19$ years old. Demographic data of the two studied groups are shown in Table I.

The NLR, albuminuria and uric acid levels of the patients with stage 3 CKD were higher than those with stages 1 and 2 ( $p=0.013, p=0.001$ and $p=$ 0.001 , respectively). However, no statistically significant difference was detected for the PLR. The results of the laboratory tests are shown in Table II.

Table I. Demographic characteristics of the two studied groups of patients according to eGFR

\begin{tabular}{|c|c|c|c|c|}
\hline Variables & $\begin{array}{l}\text { All patients } \\
\quad(n=271)\end{array}$ & $\begin{array}{c}\text { eGFR } \geq 60 \\
(n=242)\end{array}$ & $\begin{array}{c}60>\mathrm{eGFR} \geq 30 \\
(n=29)\end{array}$ & $P$-value \\
\hline Female, $n(\%)$ & $185(68.3)$ & $164(67.8)$ & $21(72.4)$ & 0.612 \\
\hline Male, $n(\%)$ & $86(31.7)$ & $78(32.2)$ & $8(27.6)$ & \\
\hline Age, mean \pm SD [years] & $63.94 \pm 11.19$ & $62.39 \pm 10.49$ & $76.90 \pm 8.14$ & $0.001^{c}$ \\
\hline Height, mean \pm SD $[\mathrm{cm}]$ & $161.26 \pm 7.67$ & $161.35 \pm 7.84$ & $160.48 \pm 6.22$ & $0.566^{c}$ \\
\hline Weight, mean \pm SD [kg] & $78.55 \pm 13.94$ & $79.00 \pm 14.01$ & $74.76 \pm 12.91$ & $0.122^{c}$ \\
\hline $\mathrm{BMI}$, mean $\pm \mathrm{SD}\left[\mathrm{kg} / \mathrm{m}^{2}\right]$ & $30.22 \pm 5.18$ & $30.35 \pm 5.16$ & $29.10 \pm 5.30$ & $0.221^{c}$ \\
\hline Systolic BP, mean \pm SD [mm Hg] & $134.38 \pm 18.73$ & $133.95 \pm 18.33$ & $137.93 \pm 21.85$ & $0.280^{c}$ \\
\hline Diastolic BP, mean \pm SD [mm Hg] & $83.41 \pm 10.16$ & $83.07 \pm 10.01$ & $86.21 \pm 11.15$ & $0.116^{c}$ \\
\hline Smoker, $n(\%)$ & $55(20.3)$ & $48(19.8)$ & $7(24.1)$ & $0.586^{\mathrm{a}}$ \\
\hline CVD, $n(\%)$ & $12(4.4)$ & $9(3.7)$ & $3(10.3)$ & $0.125^{b}$ \\
\hline CKD, $n(\%)$ & $21(7.7)$ & $11(4.5)$ & $10(34.5)$ & $0.001^{b}$ \\
\hline CAD, $n(\%)$ & $69(25.5)$ & $55(22.7)$ & $14(48.3)$ & $0.003^{a}$ \\
\hline CABG, $n(\%)$ & $12(4.4)$ & $10(4.1)$ & $2(6.9)$ & $0.624^{b}$ \\
\hline Coronary stent, $n(\%)$ & $18(6.6)$ & $16(6.6)$ & $2(6.9)$ & $1.000^{\mathrm{b}}$ \\
\hline Use of ACE and ARB, $n(\%)$ & $195(72.2)$ & $173(71.5)$ & $22(78.6)$ & $0.428^{a}$ \\
\hline Use of $\mathrm{BB}, n(\%)$ & $67(24.8)$ & $59(24.4)$ & $8(28.6)$ & $0.627^{a}$ \\
\hline Use of CCB, $n(\%)$ & $77(28.6)$ & $64(26.6)$ & $13(46.4)$ & $0.028^{a}$ \\
\hline
\end{tabular}

eGFR - estimated glomerular filtration rate $\left(\mathrm{ml} / \mathrm{min} / 1.73 \mathrm{~m}^{2}\right), B M l$ - body mass index, $B P$ - blood pressure, CVD - cerebrovascular disease, $C K D$ - chronic kidney disease, $C A D$ - coronary artery disease, $C A B G$ - coronary artery bypass graft, ACE - angiotensin converting enzyme, $A R B$ - angiotensin receptor blockers, $B B$ - $\beta$-blockers, $C C B$ - calcium channel blockers. aPearson's $\chi^{2}$ test, bFisher's exact test, 'one-way ANOVA test. 
Associations between neutrophil/lymphocyte ratio, platelet/lymphocyte ratio, albuminuria and uric acid and the estimated glomerular filtration rate in hypertensive patients with chronic kidney disease stages 1-3

Table II. Laboratory assessments of different measured variables in the two studied groups

\begin{tabular}{|c|c|c|c|c|}
\hline Variables & $\begin{array}{l}\text { All patients } \\
\quad(n=271)\end{array}$ & $\begin{array}{l}\text { eGFR } \geq 60 \\
(n=242)\end{array}$ & $\begin{array}{c}60>\mathrm{eGFR} \geq 30 \\
(n=29)\end{array}$ & $P$-value \\
\hline Glucose, mean \pm SD $[\mathrm{mg} / \mathrm{dl}]$ & $104.57 \pm 22.77$ & $104.27 \pm 20.92$ & $107.03 \pm 35.02$ & 0.538 \\
\hline Creatinine, mean $\pm \mathrm{SD}[\mathrm{mg} / \mathrm{dl}]$ & $0.83 \pm 0.19$ & $0.80 \pm 0.17$ & $1.12 \pm 0.10$ & 0.001 \\
\hline T. cholesterol, median (min.-max.) $[\mathrm{mg} / \mathrm{dl}]$ & $191(97-395)$ & $191(97-395)$ & $180(106-264)$ & 0.236 \\
\hline Triglyceride, median (min.-max.) [mg/dl] & $133(43-576)$ & $137(43-576)$ & $122(51-277)$ & 0.102 \\
\hline $\mathrm{HDL}$, mean $\pm \mathrm{SD}[\mathrm{mg} / \mathrm{dl}]$ & $45.41 \pm 9.19$ & $45.33 \pm 8.96$ & $46.07 \pm 11.08$ & 0.684 \\
\hline LDL, median (min.-max.) [mg/dl] & $113(45-294)$ & $113(46-294)$ & $106(45-196)$ & 0.668 \\
\hline Albuminuria, median (min.-max.) $[\mathrm{mg} / \mathrm{dl}]$ & $22(3.16-2446)$ & $20(3.2-694)$ & $39(5-2446)$ & 0.001 \\
\hline Uric acid, median (min.-max.) [mg/dl] & $5.6(1.3-10.8)$ & $5.4(1.3-10.8)$ & $7.1(1.3-8.9)$ & 0.001 \\
\hline Leukocytes, median (min.-max.) $\left[\times 10^{3} / \mu \mathrm{l}\right]$ & $7.2(3.42-13)$ & $7.2(3.4-13)$ & $7.5(4.3-12.1)$ & 0.161 \\
\hline Platelets, median (min.-max.) $\left[\times 10^{3} / \mu \mathrm{l}\right]$ & $229.4(94.39-454.6)$ & $230.1(94.4-454.6)$ & $214.2(104.1-352.1)$ & 0.164 \\
\hline Neutrophils, median (min.-max.) $\left[\times 10^{3} / \mu \mathrm{l}\right]$ & $4.8(1.16-10.6)$ & $4.1(1.2-10.7)$ & $4.6(2.2-9.3)$ & 0.103 \\
\hline $\begin{array}{l}\text { Lymphocytes, median (min.-max.) } \\
{\left[\times 10^{3} / \mu l\right]}\end{array}$ & $2.2(1.02-5.28)$ & $2.2(1-5.3)$ & $2.1(1.1-3.5)$ & 0.078 \\
\hline NLR, median (min.-max.) & $1.9(0.49-8.53)$ & $1.8(0.5-8.5)$ & $2.3(0.9-6.4)$ & 0.010 \\
\hline PLR, median (min.-max.) & $105.1(43.19-288.63)$ & $103.4(43.2-288.6)$ & $109.3(64.3-200.9)$ & 0.773 \\
\hline
\end{tabular}

eGFR - estimated glomerular filtration rate $\left(\mathrm{ml} / \mathrm{min} / 1.73 \mathrm{~m}^{2}\right), H D L$ - high-density lipoprotein, $L D L$ - low-density lipoprotein, NLR neutrophil/lymphocyte ratio, PLR - platelet/lymphocyte ratio. 'One-way analysis of variance.

There was a negative, weak (low $r$ ), but statistically significant correlation between the eGFR and the uric acid level $(r=-0.270, p<0.001)$ (Figure 1$)$. There was a negative, weak (low $r$ ), but statistically significant correlation between the eGFR and the albuminuria level $(r=-0.185, p=0.002)$ (Figure 2).

The results of the diagnostic tests and ROC curves for the stage 3 patients are shown in Table III.

The areas under the ROC curves were $64.3 \%$ for the NLR, $63.4 \%$ for the albuminuria and $74.3 \%$ for the uric acid. The areas under the ROC curves for

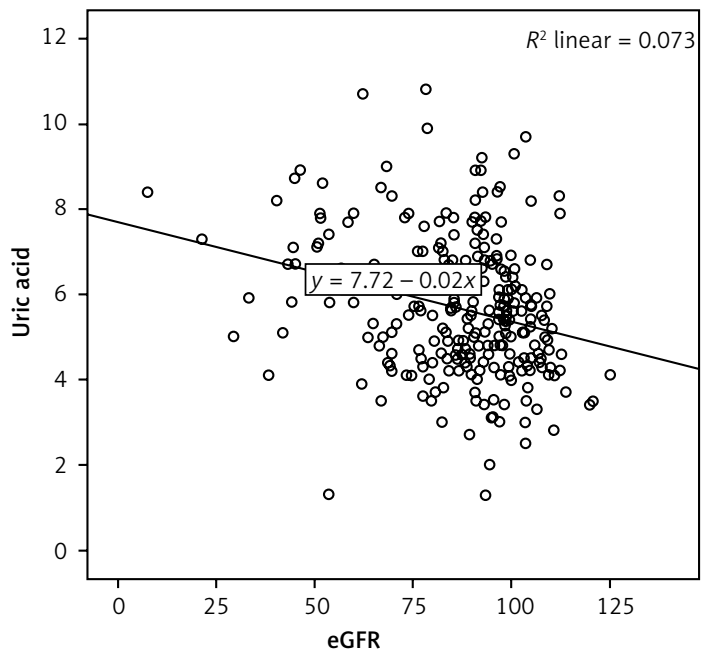

Figure 1. Correlation between eGFR and uric acid in studied patients the NLR and albuminuria were very similar to each other, whereas the area for uric acid was larger. However, no statistically significant differences were detected between the NLR and albuminuria, between the NLR and uric acid, and between albuminuria and uric acid for the areas under the ROC curves. The evaluations regarding the ROC curves are depicted in Table IV and Figure 3.

An evaluation of the risk factors affecting the eGFR, including the NLR, PLR, albuminuria and uric acid, using a stepwise logistic regression analysis revealed the model to be significant, with an ex-

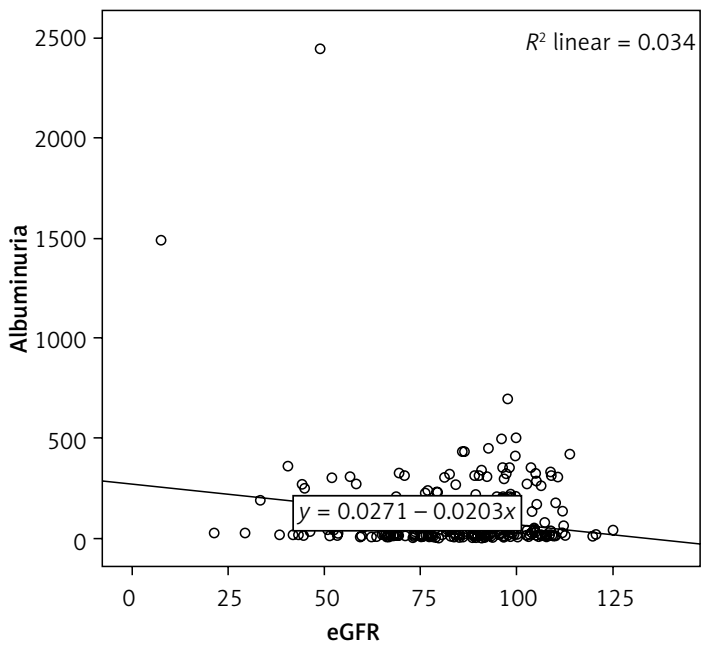

Figure 2. Correlation between eGFR and albuminuria levels in studied patients 
Table III. Diagnostic tests and ROC curve results for CKD stage 3

\begin{tabular}{|lcccccccc|}
\hline Variables & \multicolumn{4}{c}{ Importance } & \multicolumn{3}{c}{ ROC curve } & \multicolumn{2}{c|}{$\boldsymbol{P}$-value } \\
\cline { 2 - 8 } & Cut-off & Sensitivity & Specificity & PPV & NPV & Area & $95 \%$ Cl \\
\hline NLR & $>1.998$ & 62.07 & 59.09 & 15.40 & 92.90 & 0.643 & $0.583-0.700$ & 0.013 \\
\hline Albuminuria & $>25 \mathrm{mg} / \mathrm{dl}$ & 58.62 & 56.61 & 13.90 & 91.90 & 0.634 & $0.574-0.692$ & 0.009 \\
\hline Uric acid & $>6.2 \mathrm{mg} / \mathrm{dl}$ & 72.41 & 70.66 & 22.80 & 95.50 & 0.743 & $0.687-0.794$ & $<0.001$ \\
\hline
\end{tabular}

$N L R$ - neutrophil/lymphocyte ratio, PPV - positive predictive value, NPV - negative predictive value, Cl - confidence interval.

Table IV. Evaluations of NLR, PLR, albuminuria and uric acid regarding areas under ROC curves

\begin{tabular}{|c|c|c|c|c|c|}
\hline \multirow[t]{3}{*}{ Variables } & \multicolumn{5}{|c|}{ Area under the curve } \\
\hline & \multirow[t]{2}{*}{ Area } & \multirow[t]{2}{*}{ Std. error (a) } & \multirow[t]{2}{*}{$P$-value } & \multicolumn{2}{|c|}{$95 \% \mathrm{Cl}$} \\
\hline & & & & Lower & Upper \\
\hline NLR & 0.643 & 0.058 & 0.013 & 0.583 & 0.700 \\
\hline PLR & 0.526 & 0.056 & 0.644 & 0.465 & 0.587 \\
\hline Albuminuria & 0.634 & 0.052 & 0.009 & 0.574 & 0.692 \\
\hline Uric acid & 0.743 & 0.047 & $<0.001$ & 0.687 & 0.794 \\
\hline
\end{tabular}

$N L R$ - neutrophil/lymphocyte ratio, $P L R$ - platelet/lymphocyte ratio, $\mathrm{Cl}$ - confidence interval.

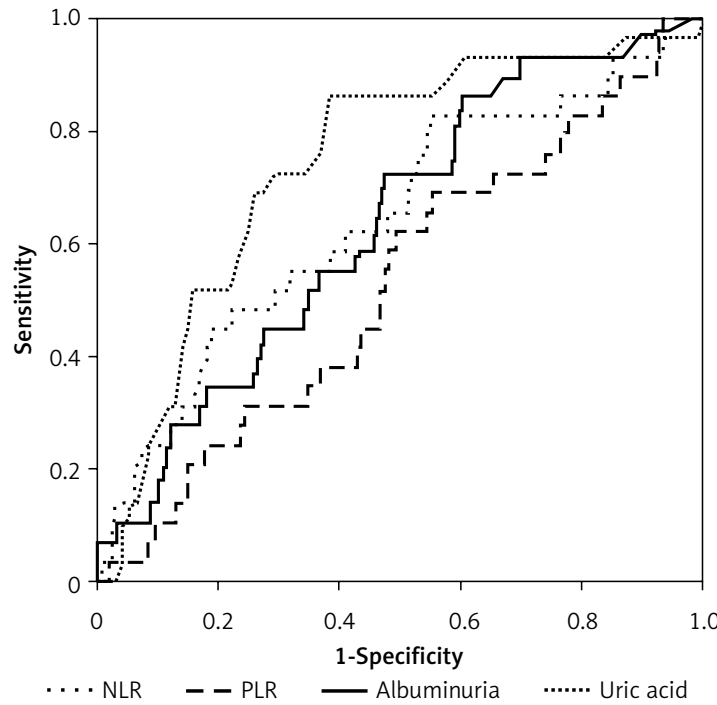

Figure 3. ROC curves for NLR, PLR, albuminuria, and uric acid planatory coefficient of $89.7 \%$. At the end of step 3, albuminuria and uric acid were found to be significant variables in the model and independent risk factors affecting the eGFR $(p<0.05)$. However, the NLR and PLR did not affect the eGFR statistically significantly. The results of the logistic regression analysis of the risk factors affecting the eGFR are shown in Table $\mathrm{V}$.

\section{Discussion}

The current study investigated the associations between the NLR and PLR and the eGFR, and their ability to serve as independent risk factors for renal damage. To the best of our knowledge, this is the first study in the literature to assess the po1.0 tentials of the NLR and PLR as predictors of essential hypertension in patients with CKD stages 1-3.

This study demonstrated higher NLR, albuminuria, and uric acid levels in the patients with stage 3 CKD than in those with stages 1 and 2. However,

Table V. Logistic regression analysis of risk factors affecting eGFR

\begin{tabular}{|lcccc|}
\hline Variables & P-value & OR & S5\% Cl \\
\cline { 3 - 4 } & & & Lower & Upper \\
\hline Albuminuria & 0.042 & 1.002 & 1.000 & 1.004 \\
\hline Uric acid & 0.001 & 1.539 & 1.204 & 1.966 \\
\hline NLR & 0.104 & 1.290 & 0.949 & 1.755 \\
\hline PLR & 0.578 & 0.996 & 0.983 & 1.009 \\
\hline
\end{tabular}

$N L R$ - neutrophil/lymphocyte ratio, $P L R$ - platelet/lymphocyte ratio, OR - odds ratio, $\mathrm{Cl}$ - confidence interval. 
no statistically significant difference was detected in the PLR. In the stage 3 patients, the albuminuria and uric acid were found to be independent risk factors affecting the eGFR, but the effects of the NLR and PLR on the eGFR were not statistically significant.

Derived from the leukocyte count, the NLR is an inexpensive, routinely used and reproducible test that has been revealed by many studies to be an indicator of the systemic inflammatory response. In addition, many studies have shown it to be associated with poor clinical outcomes in cardiac disease and several malignancies [8-11, 22, 23]. The NLR is a parameter that provides information not only about the inflammation, but also about the stress response of the patient. A high neutrophil count primarily reflects infection, while a low lymphocyte count indicates poor general health and physiological stress [10]. The blood NLR is an indicator of the general inflammatory condition and stress status of the body. A recent study reported that the NLR was associated with an increased mortality rate and poor prognosis in acute coronary syndrome, especially when ST-segment elevation is present [11]. In light of the above findings, the NLR may be a beneficial and cost-effective method to evaluate the inflammatory status. Because the inflammatory parameters and NLR are increased in diabetic patients [24, 25], we excluded diabetic patients from our study. We aimed to assess whether the NLR, an inflammatory marker in non-diabetic CKD patients, was increased or not.

A high platelet and low lymphocyte count are associated with adverse cardiovascular outcomes. For instance, Azab et al. found that a high PLR could serve as an indicator of long-term mortality in patients with acute coronary syndrome without ST-segment elevation [26]. Elevated NLR or PLR levels have been demonstrated to be associated with various adverse clinicopathological conditions in patients with certain malignancies, including ovarian cancer, colorectal cancer and pancreatic ductal adenocarcinoma [22, 23, 27]. Moreover, associations between the NLR and PLR and cardiovascular disease have been demonstrated in many studies [26, 28, 29]. For example, Sunbul et al. showed them to be significantly higher in non-dipper hypertension patients when compared to dipper hypertension patients. Furthermore, the PLR (not NLR) has been shown to be an independent predictor of non-dipper hypertension [29]. Another recent study reported the NLR to be a predictor of all-cause mortality in geriatric patients with CKD stages 3-5 [19]. Similarly, in the present study, an increased NLR was observed in the patients with CKD stage 3. The NLR level, especially in patients with values of 1.9 or more, demonstrated a high specificity in predicting a low eGFR (similar to albuminuria and uric acid). Additionally, at a cut-off of 5.8, the NLR showed a specificity of more than $99 \%$. In their study, Tatar et al. did not find a statistically significant association between the PLR and eGFR in patients with CKD stages 3-5 [19]. Similarly, we did not detect a significant association between the eGFR and PLR in patients with CKD stages 1-3.

Both microalbuminuria and macroalbuminuria are considered to be indicators of the progression of renal dysfunction, and they are still in use. They also serve as predictors of cardiovascular disease $[17,18,30]$. Especially in the geriatric population with normal renal function, the detection of microalbuminuria is indicative of silent and asymptomatic systemic vascular progression [18]. In addition, the data in the current study demonstrated that albuminuria was an independent predictor of renal damage in patients with CKD stage 3.

A serum uric acid elevation is a common manifestation of essential hypertension, and it is associated with high blood pressure [15, 31, 32]. Moreover, it is a potential factor in the development and/or progression of end-organ damage as a result of its association with the hypertensive status through several direct and indirect mechanisms [31-33]. One recent study proved that the serum uric acid was an independent factor, with a direct role in the development of CKD [34]. Similarly, we found that a serum uric acid level above $6.2 \mathrm{mg} / \mathrm{dl}$ showed high specificity as an independent predictor of renal damage.

The present study evaluated the independent predictors of eGFR in patients with CKD stage 3. At an NLR > 1.998, the sensitivity was $62.07 \%$ and the specificity was $59.09 \%$; the PPV was $15.4 \%$ and the NPV was $92.9 \%$. The areas under the ROC curves were $64.3 \%$ for the NLR, $63.4 \%$ for albuminuria and $74.3 \%$ for uric acid. The similarity of the areas under the ROC curves for the NLR and albuminuria is striking, although the area for the uric acid was higher. Moreover, albuminuria and uric acid were independent risk factors affecting the eGFR. However, the effects of the NLR and PLR on the eGFR were not statistically significant.

We included all patients with essential hypertension who were referred to the internal medicine clinic of our hospital. However, the number of enrolled females in the present study was higher than that of males (68.3\% vs. $31.7 \%)$. This may be due to greater negligence and non-compliance of males as regards seeking medical advice and consumption of medication. Moreover, women consider their illnesses to be more important than men do, and they regularly follow-up with their doctor visits more carefully than men. Furthermore, according to our hospital statistics, women 
are 3 times more likely to be examined than men. Thus, there was a gender bias in our study. In addition, it is known that oestrogen levels provide protection against inflammation and cardiovascular diseases. While higher oestrogen levels are associated with lower inflammation and a lower probability of ischemic stroke, cognitive functions are also found to be better in these patients [35, 36]. This selection bias may have affected our results. We included all essential hypertensive patients with eGFRs $\geq 30 \mathrm{ml} / \mathrm{min} / 1.73 \mathrm{~m}^{2}$ in our study. However, angiotensin receptor blockers (ARB), such as losartan, which protects patients against nephropathy, could have been listed in the exclusion criteria.

In conclusion, the NLR and PLR were not independent predictors of the eGFR and renal damage (in CKD patients who do not require renal replacement therapy). Based on the current data, future studies including more patients and different populations are warranted to evaluate the applicability of this NLR model.

\section{Conflict of interest}

The authors declare no conflict of interest.

\section{References}

1. Turak O, Ozcan F, Tok D, et al. Serum uric acid, inflammation and nondipping circadian pattern in essential hypertension. J Clin Hypertens 2013; 15: 7-13.

2. Silverstein DM. Inflammation in chronic kidney disease: role in the progression of renal and cardiovascular disease. Pediatr Nephrol 2009; 24: 1445-52.

3. García-Lafuente A, Guillamón E, Villares A, Rostagno MA, Martínez JA. Flavonoids as anti-inflammatory agents: implications in cancer and cardiovascular disease. Inflamm Res 2009; 58: 537-52.

4. Fadel FI, Elshamaa MF, Elghoroury EA, et al. Usefulness of serum procalcitonin as a diagnostic biomarker of infection in children with chronic kidney disease. Arch Med Sci Atheroscler Dis 2016; 1: 23-31.

5. Rysz-Górzyńska M, Banach M. Subfractions of high-density lipoprotein $(\mathrm{HDL})$ and dysfunctional $\mathrm{HDL}$ in chronic kidney disease patients. Arch Med Sci 2016; 12: 844-9.

6. Căpuşa C, Stefan G, Stancu S, Ilyes A, Dorobanțu N, Mircescu G. Subclinical cardiovascular disease markers and vitamin $D$ deficiency in non-dialysis chronic kidney disease patients. Arch Med Sci 2016; 12: 1015-22.

7. Guasti L, Dentali F, Castiglioni L, et al. Neutrophils and clinical outcomes in patients with acute coronary syndromes and/or cardiac revascularisation. A systematic review on more than 34000 subjects. Thromb Haemost 2011; 106: 591-9.

8. Tamhane UU, Aneja S, Montgomery D, Rogers EK, Eagle KA, Gurm HS. Association between admission neutrophil to lymphocyte ratio and outcomes in patients with acute coronary syndrome. Am J Cardiol 2008; 102: 653-7.

9. Uthamalingam S, Patvardhan EA, Subramanian S, et al. Utility of the neutrophil to lymphocyte ratio in predicting long-term outcomes in acute decompensated heart failure. Am J Cardiol 2011; 107: 433-8.
10. Gibson PH, Cuthbertson BH, Croal BL, et al. Usefulness of neutrophil/lymphocyte ratio as predictor of new-onset atrial fibrillation after coronary artery bypass grafting. Am J Cardiol 2010; 105: 186-91.

11. Park JJ, Jang HJ, Oh IY, et al. Prognostic value of neu trophil to lymphocyte ratio in patients presenting with ST-elevation myocardial infarction undergoing primary percutaneous coronary intervention. Am J Cardiol 2013; 111: 636-42.

12. Tsiara S, Elisaf M, Jagroop IA, Mikhailidis DP. Platelets as predictor of vascular risk: is there a practical index of platelet activity? Clin Appl Thromb Hemost 2003; 9: 177-90.

13. Dohi Y. The first step aiming at the prevention of hypertension and atherosclerosis. Identification of individuals at high risk of hypertension. Rinsho Byori 2015; 63: 1303-9.

14. Biyik I, Uzun F, Erturk M, et al. Does contrast media volume affect long-term survival in patients with chronic kidney disease? Arch Med Sci Atheroscler Dis 2017; 9: e82-9.

15. Borghi C, Rosei EA, Bardin T, et al. Serum uric acid and the risk of cardiovascular and renal disease. J Hypertens 2015; 33: 1729-41.

16. Maloberti A, Maggioni S, Occhi L, et al. Sex-related relationships between uric acid and target organ damage in hypertension. J Clin Hypertens 2017; 20: 193-200.

17. Gerstein HC, Mann JF, Yi Q, et al. Albuminuria and risk of cardiovascular events, death and heart failure in diabetic and nondiabetic individuals. JAMA 2001; 286: 421-6.

18. Xiang Lei K, Xiao Yan J, Yong W, et al. Association between microalbuminuria and subclinical atherosclerosis evaluated by carotid artery intima-media in elderly patients with normal renal function. BMC Nephrol 2012; 13: 37.

19. Tatar E, Mirili C, Isikyakar T, et al. The association of neutrophil/lymphocyte ratio and platelet/lymphocyte ratio with clinical outcomes in geriatric patients with stage 3-5 chronic kidney disease. Acta Clin Belg 2016; 71: 221-6.

20. Pickering TG, Hall JE, Appel LJ, et al. Recommendations for blood pressure measurement in humans and experimental animals: part 1: blood pressure measurement in humans: a statement for professionals from the Subcommittee of Professional and Public Education of the American Heart Association Council on High Blood Pressure Research. Circulation 2005; 111: 697-716.

21. Levey AS, Stevens LA, Schmid $\mathrm{CH}$, et al. A new equation to estimate glomerular filtration rate. Ann Intern Med 2009; 150: 604-12.

22. Halazun KJ, Aldoori A, Malik HZ, et al. Elevated preoperative neutrophil to lymphocyte ratio predicts survival following hepatic resection for colorectal liver metastases. Eur J Surg Oncol 2008; 34: 55-60.

23. Asher V, Lee J, Innamaa A, Bali A. Preoperative platelet lymphocyte ratio as an independent prognostic marker in ovarian cancer. Clin Transl Oncol 2011; 13: 499-503.

24. Wellen KE, Hotamisligil GS. Inflammation, stress and diabetes. J Clin Invest 2005; 115: 1111-9.

25. Kahraman C, Kahraman NK, Aras B, Coşgun S, Gülcan E. The relationship between neutrophil-to-lymphocyte ratio and albuminuria in type 2 diabetic patients: a pilot study. Arch Med Sci 2016; 12: 571-5.

26. Azab B, Shah N, Akerman M, McGinn Jr JT. Value of platelet/lymphocyte ratio as a predictor of all-cause mortality after non-ST elevation myocardial infarction. J Thromb Thrombolysis 2012; 34: 326-34. 
27. Bhatti I, Peacock O, Lloyd G, Larvin M, Hall RI. Preoperative hematologic markers as independent predictors of prognosis in resected pancreatic ductal adenocarcinoma: neutrophil-lymphocyte versus platelet-lymphocyte ratio. Am J Surg 2010; 200: 197-203.

28. Demir M. The relationship between neutrophil lymphocyte ratio and nondipper hypertension. Clin Exp Hypertens 2013; 35: 570-3.

29. Sunbul M, Gerin F, Durmus E, et al. Neutrophil to lymphocyte and platelet to lymphocyte ratio in patients with dipper versus non-dipper hypertension. Clin Exp Hypertens 2014; 36: 217-21.

30. Verhave JC, Gansevoort RT, Hillege HL, Bakker SJ, De Zeeuw D. An elevated urinary albumin excretion predicts de novo development of renal function impairment in the general population. Kidney Int 2004; 92: 18-21.

31. Viazzi F, Leoncini G, Ratto E, et al. Serum uric acid as risk factor for cardiovascular and renal disease: an old controversy revived. J Clin Hypertens 2006; 5: 510-8.

32. Agabiti-Rosei E, Grassi G. Beyond gout: uric acid and cardiovascular diseases. Curr Med Res Opin 2013; 3 29 Suppl: 33-9.

33. Cuspidi C, Valerio C, Sala C, et al. Lack of association between serum uric acid and organ damage in a never-treated essential hypertensive population at low prevalence of hyperuricemia. Am J Hypertens 2007; 20: 678-85.

34. Maloberti A, Maggioni S, Occhi L, et al. Sex-related relationships between uric acid and target organ damage in hypertension. J Clin Hypertens (Greenwich) 2018; 20: 193-200.

35. Hsieh YC, Hsieh FI, Chen YR, et al. Associations of estradiol levels and genetic polymorphisms of inflammatory genes with the risk of ischemic stroke. J Biomed Sci 2017; 24: 25.

36. Au A, Feher A, McPhee L, et al. Estrogens, inflammation and cognition. Front Neuroendocrinol 2016; 40: 87-100. 